\title{
On Gap Functions for Quasi-Variational Inequalities
}

\begin{abstract}
Kouichi Taji
Department of Mechanical Science and Engineering, Graduate School of Engineering, Nagoya University,
\end{abstract} Furo-cho, Chikusa, Nagoya 464-8603, Japan

Correspondence should be addressed to Kouichi Taji, taji@nuem.nagoya-u.ac.jp

Received 14 September 2007; Revised 18 December 2007; Accepted 8 January 2008

Recommended by Nobuyuki Kenmochi

For variational inequalities, various merit functions, such as the gap function, the regularized gap function, the D-gap function and so on, have been proposed. These functions lead to equivalent optimization formulations and are used to optimization-based methods for solving variational inequalities. In this paper, we extend the regularized gap function and the D-gap functions for a quasi-variational inequality, which is a generalization of the variational inequality and is used to formulate generalized equilibrium problems. These extensions are shown to formulate equivalent optimization problems for quasi-variational inequalities and are shown to be continuous and directionally differentiable.

Copyright (C) 2008 Kouichi Taji. This is an open access article distributed under the Creative Commons Attribution License, which permits unrestricted use, distribution, and reproduction in any medium, provided the original work is properly cited.

\section{Introduction}

In this paper, we consider merit functions for quasi-variational inequalities, denoted by QVIP, to find a vector $x^{*} \in S\left(x^{*}\right)$ such that

$$
\left\langle F\left(x^{*}\right), x-x^{*}\right\rangle \geq 0 \quad \forall x \in S\left(x^{*}\right),
$$

where $F: \mathbb{R}^{n} \rightarrow \mathbb{R}^{n}$ is a mapping, the symbol $\langle\cdot, \cdot\rangle$ denotes the inner product in $\mathbb{R}^{n}$, and $S$ : $\mathbb{R}^{n} \rightarrow 2^{\mathbb{R}^{n}}$ is a set-valued mapping of which $S(x)$ is a closed convex set in $\mathbb{R}^{n}$ for each $x$. When the mapping $S$ is a constant closed convex set for all $x \in \mathbb{R}^{n}$, QVIP reduces to a well-known variational inequality $[1,2]$ :

$$
\left\langle F\left(x^{*}\right), x-x^{*}\right\rangle \geq 0 \quad \forall x \in S .
$$

QVIP is used to study and formulate generalized equilibrium problems, such as the generalized Nash equilibrium problem in which a strategy set of each player varies according to 
the other players' strategies $[3,4]$. For variational inequalities, various merit functions, such as the gap function, the regularized gap function [5], the D-gap functions [6, 7], and so on, have been proposed. These functions are used to make an equivalent optimization formulation for a variational inequality, and based on these formulations, several optimization-based methods are proposed for solving variational inequalities $[8,9]$.

Fukushima [10] has proposed gap functions for QVIP, which lead to equivalent optimization formulation for QVIP. In this paper, we extend the results of Fukushima in two directions. One is to show the directional differentiability under more general setting (Theorem 2.5) and to give one sufficient condition for stationary point to be a solution (Proposition 2.7). The other is to propose a so-called D-gap function for QVIP, which leads to an unconstrained minimization optimization formulation for QVIP, and to show its directional differentiability (Section 3).

Notations used in this paper are summarized as follows. The superscript $T$ denotes a transpose of vector or matrix, and $\|\cdot\|$ denotes the Euclidean norm in $\mathbb{R}^{n}$ defined by $\|x\|=$ $\sqrt{\langle x, x\rangle}$.

\section{Regularized gap function for QVIP}

In this section, we first generalize the regularized gap function for a variational inequality to a quasi-variational inequality and show its properties.

The (generalized) regularized gap functions $f_{\alpha}: \mathbb{R}^{n} \rightarrow \mathbb{R}$ for QVIP (1.1) are defined as

$$
f_{\alpha}(x)=\max _{y}\{-\langle F(x), y-x\rangle-\alpha \phi(x, y) \mid y \in S(x)\},
$$

where an $\alpha$ is a positive constant and a function $\phi: \mathbb{R}^{n} \times \mathbb{R}^{n} \rightarrow \mathbb{R}$ satisfies the following conditions.

(C1) $\phi$ is continuously differentiable on $\mathbb{R}^{n} \times \mathbb{R}^{n}$.

(C2) $\phi$ is nonnegative on $\mathbb{R}^{n} \times \mathbb{R}^{n}$ and $\phi(x, y)=0$ if and only if $x=y$.

(C3) $\phi$ is strongly convex uniformly in $x$, that is, there is a $\mu>0$ such that

$$
\phi\left(x, y_{1}\right)-\phi\left(x, y_{2}\right) \geq\left\langle\nabla_{y} \phi\left(x, y_{2}\right), y_{1}-y_{2}\right\rangle+\mu\left\|y_{1}-y_{2}\right\|^{2} \quad \forall y_{1}, y_{2} \in \mathbb{R}^{n} .
$$

Remark 2.1. It is easy to verify that a function $\phi(x, y)=\langle x-y, G(x-y)\rangle$ for a positive definite symmetric matrix $G$ satisfies the above conditions $(\mathrm{C} 1) \sim(\mathrm{C} 3)$. In this case, the function (2.1) reduces to

$$
f(x)=\max _{y}\left\{-\langle F(x), y-x\rangle-\frac{1}{2}\langle y-x, G(y-x)\rangle \mid y \in S(x)\right\}
$$

with $\alpha=1 / 2$. This is just a regularized gap function [5] originally proposed for variational inequalities (1.2).

By the strong convexity of $\phi$ and the closed convexity of $S(x)$, the maximum in (2.1) is uniquely attained and is given by the unique solution $y_{\alpha}(x)$ of the following mathematical programming problem:

$$
\min _{y}\langle F(x), y-x\rangle+\alpha \phi(x, y) \quad \text { s.t. } y \in S(x),
$$

and the function (2.1) is written as

$$
f_{\alpha}(x)=-\left\langle F(x), y_{\alpha}(x)-x\right\rangle-\alpha \phi\left(x, y_{\alpha}(x)\right) \text {. }
$$


Lemma 2.2. A point $x^{*}$ is a solution to QVIP (1.1) if and only if $y_{\alpha}\left(x^{*}\right)=x^{*}$.

Proof. Since the optimization problem (2.4) is convex with respect to $y$, the point $y_{\alpha}\left(x^{*}\right)$ is a solution to (2.4) if and only if

$$
\left\langle F\left(x^{*}\right)+\alpha \nabla_{y} \phi\left(x^{*}, y\right), y-y_{\alpha}\left(x^{*}\right)\right\rangle \geq 0 \quad \forall y \in S\left(x^{*}\right),
$$

which is equivalent to

$$
\left\langle\alpha \nabla_{y} \phi\left(x^{*}, y\right), y-y_{\alpha}\left(x^{*}\right)\right\rangle \geq\left\langle F\left(x^{*}\right), y_{\alpha}\left(x^{*}\right)-y\right\rangle .
$$

Then by substituting a solution $x^{*}$ of QVIP (1.1) to $y$, we have

$$
\left\langle\nabla_{y} \phi\left(x^{*}, y\right), x^{*}-y_{\alpha}\left(x^{*}\right)\right\rangle \geq 0 .
$$

On the other hand, from the condition (C3) we have

$$
\left\langle\nabla_{y} \phi\left(x^{*}, y_{\alpha}\left(x^{*}\right)\right), x^{*}-y_{\alpha}\left(x^{*}\right)\right\rangle+\mu\left\|x^{*}-y_{\alpha}\left(x^{*}\right)\right\|^{2} \leq \phi\left(x^{*}, x^{*}\right)-\phi\left(x^{*}, y_{\alpha}\left(x^{*}\right)\right) \leq 0,
$$

where the last inequality follows from the condition (C2). The above two inequalities lead to $y_{\alpha}\left(x^{*}\right)=x^{*}$.

Conversely, suppose that $y_{\alpha}\left(x^{*}\right)=x^{*}$. Then the inequality (2.6) reduces to

$$
\left\langle F\left(x^{*}\right), y-x^{*}\right\rangle \geq 0 \quad \forall y \in S\left(x^{*}\right),
$$

which shows that $x^{*}$ is a solution to (1.1).

The next theorem shows that the function (2.1) or (2.5) leads to an equivalent optimization problem for quasi-variational inequalities. The theorem is inherently equivalent to [10, Theorem 2], but for completeness, we provide its proof. We note that our proof is more elementary and simpler than that of [10, Theorem 2].

Theorem 2.3. Let $f_{\alpha}$ be the function defined by (2.1) or (2.5). Then $f_{\alpha}(x) \geq 0$ for all $x \in S(x)$. Furthermore, $f_{\alpha}\left(x^{*}\right)=0$ and $x^{*} \in S\left(x^{*}\right)$ if and only if $x^{*}$ is a solution to QVIP (1.1). Hence, problem (1.1) is equivalent to finding a global optimal solution to the problem:

$$
\text { minimize } f_{\alpha}(x) \text { subject to } x \in S(x) \text {. }
$$

Proof. The first assertion is obvious from the definition (2.1) and (C2). To prove the last assertion, suppose that $x^{*}$ is a solution to QVIP. Then, we have

$$
\left\langle F\left(x^{*}\right), x^{*}-x\right\rangle-\alpha \phi\left(x^{*}, y\right) \leq 0 \quad \forall y \in S\left(x^{*}\right) .
$$

Therefore, from the definition (2.1), we have $f_{\alpha}\left(x^{*}\right)=0$.

For the "only if" part, we consider the regularized gap function for fixed $x^{*}$,

$$
\widehat{f}_{\alpha}(x)=\max _{y}\left\{-\langle F(x), y-x\rangle-\alpha \phi(x, y) \mid y \in S\left(x^{*}\right)\right\} .
$$

Then, it follows from $f_{\alpha}\left(x^{*}\right)=0$ and $x^{*} \in S\left(x^{*}\right)$ that $\widehat{f}_{\alpha}\left(x^{*}\right)=0$, which implies that $x^{*}$ is a solution to the variational inequality [11]

$$
\left\langle F\left(x^{*}\right), x-x^{*}\right\rangle \geq 0 \quad \forall x \in S\left(x^{*}\right) .
$$

This means that $x^{*}$ is a solution to QVIP. 
The next theorem gives a sufficient condition for the continuity of the function (2.1).

Theorem 2.4. Let $f_{\alpha}$ be the function defined by (2.1) or (2.5). If the set-valued mapping $S$ is continuous with respect to $x$ in the sense of set-valued mapping [12], then $f_{\alpha}$ is also continuous in $x$.

Proof. In a similar way to [13], $y_{\alpha}$ is shown to be continuous in $x$. Therefore, the function $f_{\alpha}$ is also continuous. such that

When the set-valued mapping $S$ is expressed as a finite number of convex inequalities,

$$
S(x)=\left\{y \in \mathbb{R}^{n} \mid g_{i}(x, y) \leq 0, i=1, \ldots, m\right\},
$$

where the functions $g_{i}(x, y): \mathbb{R}^{n} \times \mathbb{R}^{n} \rightarrow \mathbb{R}$ are continuous with respect to $x$ and $y$, and $g_{i}(x, \cdot)$ are convex for each $x$, then one sufficient condition for the continuity of the set-valued mapping $S$ is that Slater's constraint qualification holds, that is, for each $x$, there exists a vector $\widehat{y}$ (possibly depending on $x$ ) such that

$$
g_{i}(x, \widehat{y})<0 \quad \forall i=1, \ldots, m
$$

In this case, $f_{\alpha}$ is also continuous and $y_{\alpha}(x)$ satisfies the KKT condition:

$$
\begin{gathered}
F(x)+\alpha \nabla_{y} \phi\left(x, y_{\alpha}(x)\right)+\sum_{i=1}^{m} \lambda_{i} \nabla_{y} g_{i}\left(x, y_{\alpha}(x)\right)=0 \\
g_{i}\left(x, y_{\alpha}(x)\right) \leq 0, \quad \lambda_{i} \geq 0, \quad \lambda_{i} g_{i}\left(x, y_{\alpha}(x)\right)=0, \quad i=1, \ldots, m .
\end{gathered}
$$

Unfortunately, the function $f_{\alpha}$ defined by (2.1) or (2.5) is not necessarily differentiable. However, the next theorem gives one sufficient condition of the directional differentiability of the function $f_{\alpha}$ with the set $S$ given by (2.15).

Theorem 2.5. Let the mapping $F: \mathbb{R}^{n} \rightarrow \mathbb{R}^{n}$ be continuously differentiable. Let also the set-valued mapping $S$ be defined as (2.15), where the functions $g_{i}(x, y): \mathbb{R}^{n} \times \mathbb{R}^{n} \rightarrow \mathbb{R}$ are continuous with respect to $x$ and $y$, and $g_{i}(x, \cdot)$ is convex for each $x$. If Slater's constraint qualification (2.16) holds, then the function $f_{\alpha}$ defined by (2.1) or (2.5) is directionally differentiable in any direction $d \in \mathbb{R}^{n}$, and its directional derivative $f_{\alpha}^{\prime}(x ; d)$ is given by

$$
f_{\alpha}^{\prime}(x ; d)=\min _{\lambda \in M(x)}\left(F(x)+\nabla F(x)\left(x-y_{\alpha}(x)\right)-\alpha \nabla_{x} \phi\left(x, y_{\alpha}(x)\right)-\sum_{i=1}^{m} \lambda_{i} \nabla_{x} g_{i}\left(x, y_{\alpha}(x)\right)\right)^{T} d
$$

where $M(x)$ is defined by

$$
M(x)=\left\{\lambda \in \mathbb{R}^{m} \mid \begin{array}{c}
F(x)+\alpha \nabla_{y} \phi\left(x, y_{\alpha}(x)\right)+\sum_{i=1}^{m} \lambda_{i} \nabla_{y} g_{i}\left(x, y_{\alpha}(x)\right)=0 \\
\lambda_{i} \geq 0, \lambda_{i} g_{i}\left(x, y_{\alpha}(x)\right)=0, i=1, \ldots, m
\end{array}\right\}
$$


Proof. This directly follows from [14, Theorem 2].

Remark 2.6. Fukushima [10, Theorem 3] has also proven the directional differentiability of $f_{\alpha}$ for the function (2.3) with $S(x)$ polyhedral convex. This situation is a special case of this theorem.

The next proposition gives a sufficient condition for a stationary point of the equivalent optimization problem (2.11) to be a solution of QVIP (1.1) with the set $S$ given by (2.15).

Proposition 2.7. Suppose that $x \in S(x)$. Suppose also that $\nabla F(x)$ is positive definite and $\lambda_{i}\left\langle\nabla_{x} g_{i}\left(x, y_{\alpha}(x)\right), y_{\alpha}(x)-x\right\rangle \geq 0$ for all $i$ and $\lambda \in M(x)$. If the function $\phi$ used in the regularized gap function (2.1) or (2.5) is $\phi(x, y)=\tilde{\phi}(x-y)$, where $\tilde{\phi}: \mathbb{R}^{n} \rightarrow \mathbb{R}$ is differentiable strongly convex function, then a stationary point $x$ of the problem (2.11) is a solution to QVIP (1.1).

Proof. It suffices to show that $x$ is a solution to QVIP if the following inequality holds:

$$
f^{\prime}\left(x ; y_{\alpha}(x)-x\right) \geq 0 .
$$

It is easy to see that $\phi(x, y)=\tilde{\phi}(x-y)$ satisfies the conditions $(\mathrm{C} 1) \sim(\mathrm{C} 3)$ and that $\nabla_{x} \phi(x-y)=-\nabla_{y} \phi(x-y)$. Then from the definition of directional derivative (2.18) and the KKT condition (2.17) for $y_{\alpha}(x)$, we have

$$
\begin{aligned}
f^{\prime}\left(x ; y_{\alpha}(x)-x\right) & \\
= & \min _{\lambda \in M(x)}\left(F(x)+\nabla F(x)\left(x-y_{\alpha}(x)\right)-\alpha \nabla_{x} \phi\left(x, y_{\alpha}(x)\right)-\sum_{i=1}^{m} \lambda_{i} \nabla_{x} g_{i}\left(x, y_{\alpha}(x)\right)\right)^{T}\left(y_{\alpha}(x)-x\right) \\
= & -\left(y_{\alpha}(x)-x\right)^{T} \nabla F(x)\left(y_{\alpha}(x)-x\right)-\left(\sum_{i=1}^{m} \lambda_{i} \nabla_{y} g_{i}\left(x, y_{\alpha}(x)\right)\right)^{T}\left(y_{\alpha}(x)-x\right) \\
& -\max _{\lambda \in M(x)}\left(\sum_{i=1}^{m} \lambda_{i} \nabla_{x} g_{i}\left(x, y_{\alpha}(x)\right)\right)^{T}\left(y_{\alpha}(x)-x\right) .
\end{aligned}
$$

Since functions $g_{i}$ are convex with respect to $y$, we have

$$
g_{i}(x, x)-g_{i}\left(x, y_{\alpha}(x)\right) \geq \nabla_{y} g_{i}\left(x, y_{\alpha}(x)\right)^{T}\left(x-y_{\alpha}(x)\right) .
$$

Then we have from the fact $x \in S$ and (2.17) that

$$
\begin{aligned}
-\left(\sum_{i=1}^{m} \lambda_{i} \nabla_{y} g_{i}\left(x, y_{\alpha}(x)\right)\right)^{T}\left(y_{\alpha}(x)-x\right) & \leq \sum_{i=1}^{m} \lambda_{i}\left(g_{i}(x, x)-g_{i}\left(x, y_{\alpha}(x)\right)\right. \\
& \leq-\sum_{i=1}^{m} \lambda_{i} g_{i}\left(x, y_{\alpha}(x)\right) \leq 0 .
\end{aligned}
$$

Therefore, it follows from (2.21) and the assumption $\lambda_{i}\left\langle\nabla_{x} g_{i}\left(x, y_{\alpha}(x)\right), y_{\alpha}(x)-x\right\rangle \geq 0$ that

$$
\begin{aligned}
0 & \leq f^{\prime}\left(x ; y_{\alpha}(x)-x\right) \\
& \leq-\left(y_{\alpha}(x)-x\right)^{T} \nabla F(x)\left(y_{\alpha}(x)-x\right)-\max _{\lambda \in M(x)}\left(\sum_{i=1}^{m} \lambda_{i} \nabla_{x} g_{i}\left(x, y_{\alpha}(x)\right)\right)^{T}\left(y_{\alpha}(x)-x\right) \\
& \leq-\left(y_{\alpha}(x)-x\right)^{T} \nabla F(x)\left(y_{\alpha}(x)-x\right),
\end{aligned}
$$


which leads to $y_{\alpha}(x)=x$ from the positive definiteness of $\nabla F$. This shows from Lemma 2.2 that $x$ is a solution to QVIP.

Remark 2.8. When the functions $g_{i}$ are all defined as linearized approximation of convex functions $c_{i}: \mathbb{R}^{n} \rightarrow \mathbb{R}$ at $x$, that is, $g_{i}(x, y)=c_{i}(x)+\nabla c_{i}(x)^{T}(y-x)$, then we have

$$
\nabla_{x} g_{i}\left(x, y_{\alpha}(x)\right)=\nabla c_{i}(x)+\nabla^{2} c_{i}(x)\left(y_{\alpha}(x)-x\right)-\nabla c_{i}(x)=\nabla^{2} c_{i}(x)\left(y_{\alpha}(x)-x\right) .
$$

Since the Hesse matrix $\nabla^{2} c_{i}(x)$ is positive semidefinite from the convexity of $c_{i}$, the assumption of Proposition 2.7 is satisfied. This result has been already obtained by Taji and Fukushima [13] for this setting, and the above proposition is considered as a generalization in some sense.

\section{D-gap function for QVIP}

For $0<\alpha<\beta$, we consider the function $g_{\alpha \beta}$ defined by

$$
\Phi_{\alpha \beta}(x)=f_{\alpha}(x)-f_{\beta}(x) .
$$

This is a so-called D-gap function and is originally introduced for the variational inequality (1.2) by Peng [6]. D-gap functions are shown to construct a differentiable equivalent unconstrained optimization formulation for VIPs.

We have the next proposition.

Proposition 3.1. For $0<\alpha<\beta$, for the function $\Phi_{\alpha \beta}$ defined by (3.1),

$$
(\beta-\alpha) \phi\left(x, y_{\beta}(x)\right) \leq \Phi_{\alpha \beta}(x) \leq(\beta-\alpha) \phi\left(x, y_{\alpha}(x)\right) .
$$

Proof. From the definition (3.1) and the fact that $y_{\alpha}(x), y_{\beta} \in S(x)$, we have

$$
\begin{aligned}
\Phi_{\alpha \beta}(x) & =\max _{y \in S(x)}\{-\langle F(x), y-x\rangle-\alpha \phi(x, y)\}-\max _{y \in S(x)}\{-\langle F(x), y-x\rangle-\beta \phi(x, y)\} \\
& \geq\left\langle F(x), x-y_{\beta}(x)\right\rangle-\alpha \phi\left(x, y_{\beta}(x)\right)-\left\langle F(x), x-y_{\beta}(x)\right\rangle+\beta \phi\left(x, y_{\beta}(x)\right) \\
& =(\beta-\alpha) \phi\left(x, y_{\beta}(x)\right) .
\end{aligned}
$$

This shows the left-side hand of the inequality. The right-hand side is shown in a similar way.

This proposition establishes the equivalence between a QVIP and the unconstrained minimization of a D-gap function $\Phi_{\alpha \beta}$.

Theorem 3.2. Let the function $\Phi_{\alpha \beta}$ be defined as (3.1). Then, for $0<\alpha<\beta, \Phi_{\alpha \beta}(x) \geq 0$ for all $x \in \mathbb{R}^{n}$. Moreover, $\Phi_{\alpha \beta}(x)=0$ if and only if $x$ is a solution to QVIP. Hence, the problem (1.1) is equivalent to finding a global optimal solution to the unconstrained minimization problem:

$$
\text { minimize } \Phi_{\alpha \beta}(x) \text {. }
$$

Proof. The first half follows directly from Proposition 3.1. The last half also follows from Lemma 2.2 and Proposition 3.1. 
The continuity and the directional differentiability of the D-gap function (3.1) directly follow from those of the regularized gap function (2.1).

Theorem 3.3. Suppose that the set-valued mapping $S$ is continuous in $x$, then the D-gap function $\Phi_{\alpha \beta}$ defined by (3.1) is continuous in $x$. Moreover, if the set-valued mapping $S$ is defined by (2.15) and if Slater's constraint qualification (2.16) holds, then the D-gap function $\Phi_{\alpha \beta}$ is directionally differentiable in all direction $d$.

Proof. These results directly follow from the definition of the D-gap function $\Phi_{\alpha \beta}$ and Theorems 2.4 and 2.5 .

\section{Acknowledgment}

The author would like to thank an anonymous referee for introducing the paper [10].

\section{References}

[1] F. Facchinei and J.-S. Pang, Finite-Dimensional Variational Inequalities and Complementarity Problems. Vol. I, Springer Series in Operations Research, Springer, New York, NY, USA, 2003.

[2] F. Facchinei and J.-S. Pang, Finite-Dimensional Variational Inequalities and Complementarity Problems. Vol. II, Springer Series in Operations Research, Springer, New York, NY, USA, 2003.

[3] P. T. Harker, "Generalized Nash games and quasi-variational inequalities," European Journal of Operational Research, vol. 54, no. 1, pp. 81-94, 1991.

[4] J.-S. Pang and M. Fukushima, "Quasi-variational inequalities, generalized Nash equilibria, and multileader-follower games," Computational Management Science, vol. 2, no. 1, pp. 21-56, 2005.

[5] M. Fukushima, "Equivalent differentiable optimization problems and descent methods for asymmetric variational inequality problems," Mathematical Programming, vol. 53, no. 1, pp. 99-110, 1992.

[6] J.-M. Peng, "Equivalence of variational inequality problems to unconstrained minimization," Mathematical Programming, vol. 78, no. 3, pp. 347-355, 1997.

[7] N. Yamashita, K. Taji, and M. Fukushima, “Unconstrained optimization reformulations of variational inequality problems," Journal of Optimization Theory and Applications, vol. 92, no. 3, pp. 439-456, 1997.

[8] K. Taji, M. Fukushima, and T. Ibaraki, "A globally convergent Newton method for solving strong monotone variational inequalities," Mathematical Programming, vol. 58, no. 3, pp. 369-383, 1993.

[9] J.-M. Peng and M. Fukushima, "A hybrid Newton method for solving the variational inequality problem via the D-gap function," Mathematical Programming, vol. 86, no. 2, pp. 367-386, 1999.

[10] M. Fukushima, "A class of gap functions for quasi-variational inequality problems," Journal of Industrial and Management Optimization, vol. 3, no. 2, pp. 165-171, 2007.

[11] J. H. Wu, M. Florian, and P. Marcotte, "A general descent framework for the monotone variational inequality problem," Mathematical Programming, vol. 61, no. 3, pp. 281-300, 1993.

[12] W. Hogan, "Point-to-set maps in mathematical programming," SIAM Review, vol. 15, pp. 591-603, 1973.

[13] K. Taji and M. Fukushima, "A new merit function and a successive quadratic programming algorithm for variational inequality problems," SIAM Journal on Optimization, vol. 6, no. 3, pp. 704-713, 1996.

[14] W. Hogan, "Directional derivatives for extremal-value functions with applications to the completely convex case," Operations Research, vol. 21, pp. 188-209, 1973. 


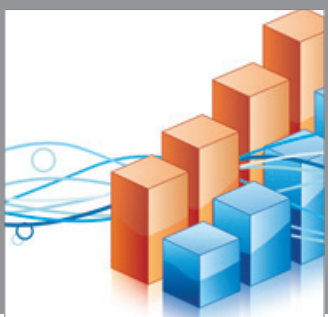

Advances in

Operations Research

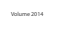

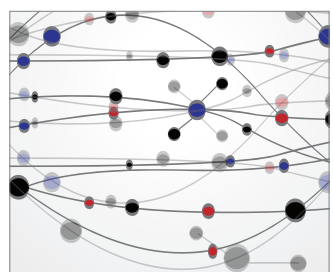

\section{The Scientific} World Journal
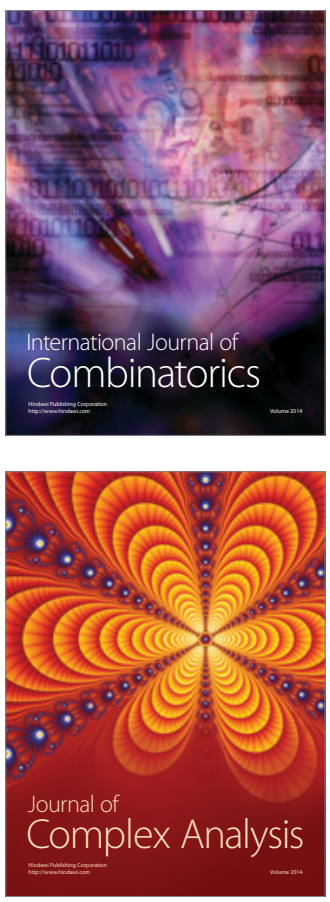

International Journal of

Mathematics and

Mathematical

Sciences
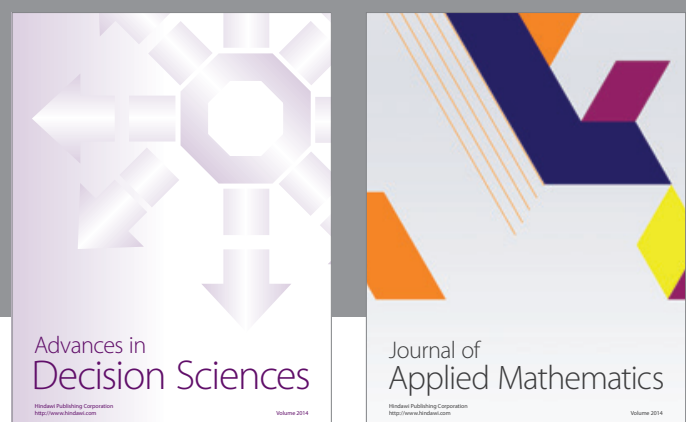

Journal of

Applied Mathematics
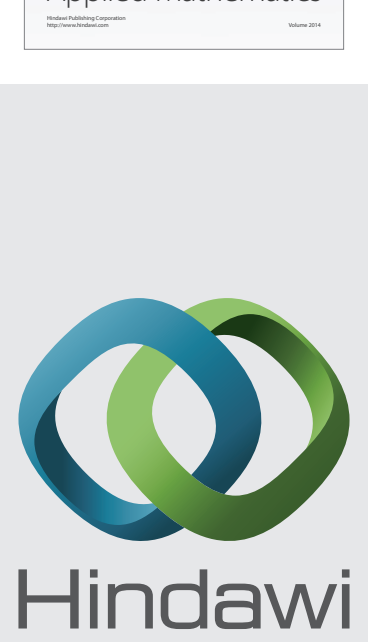

Submit your manuscripts at http://www.hindawi.com
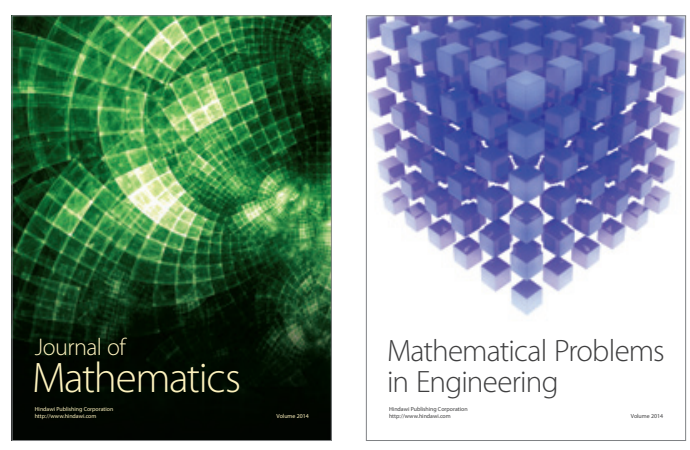

Mathematical Problems in Engineering
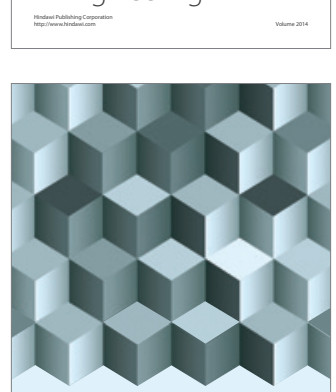

Journal of

Function Spaces
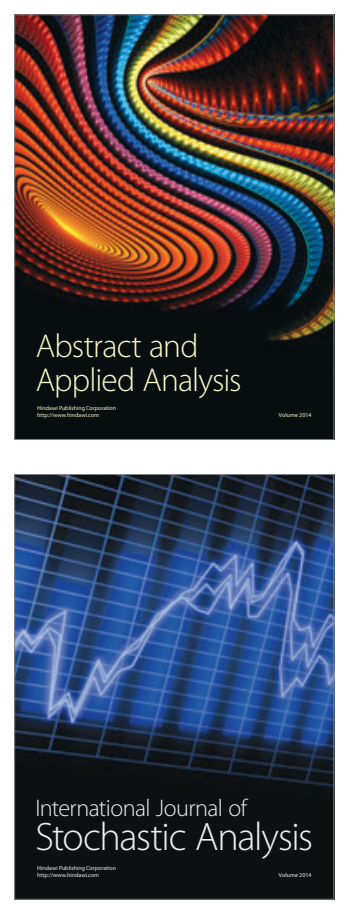

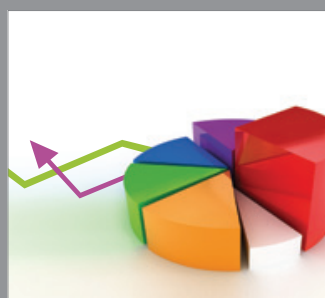

ournal of

Probability and Statistics

Promensencen
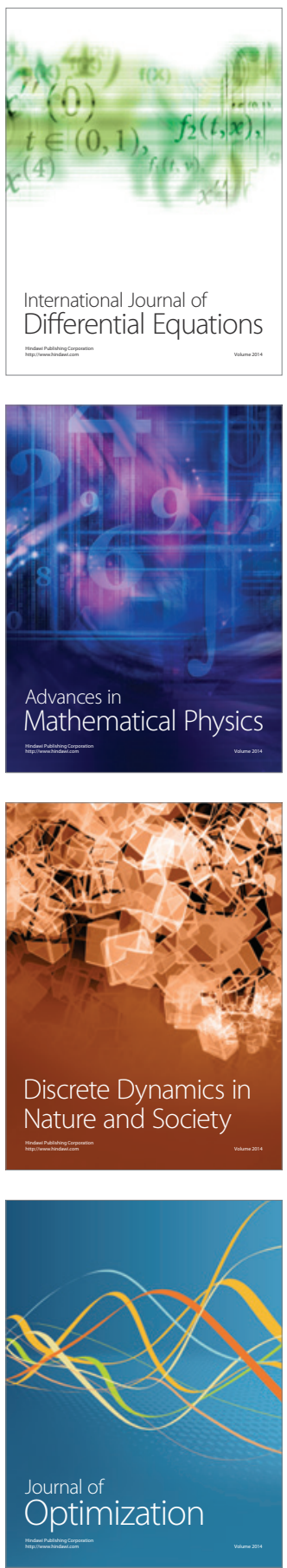